[5] W. H. Kwon and A. E. Pearson, "A Note on Feedback Stabilization of a Differential-Difference System," IEEE Trans. Automat. Contr., vol. AC-22, pp. $468-470,1977$.

[6] A. Feliachi and A. Thowsen, "Memoryless stabilization of linear delaydifferential systems," IEEE Trans. Automat. Contr., vol. AC-26, pp 586-587, 1981.

[7] E. Kreindle and A. Jameson, "Conditions for nonnegativeness of partitioned matrices," IEEE Trans. Automat. Contr., vol. AC-17, pp. $147-148,1972$

\section{Guardian Map Approach to Robust Stability of Linear Systems with Constant Real Parameter Uncertainty}

Shuoh Rern, Pierre T. Kabamba, and Dennis S. Bernstein

\begin{abstract}
New sufficient conditions are derived for stability robustness of linear time-invariant state-space systems with constant real parameter uncertainty. These bounds are obtained by applying a guardian map to the uncertain system matrices. Since this approach is only valid for constant real parameter uncertainty, these bounds do not imply quadratic stability, which guarantees robust stability with respect to timevarying uncertainty but is often conservative with respect to constant real parameter uncertainty. Numerical results are given to compare the new bounds with bounds obtained previously by means of Lyapunov methods.
\end{abstract}

\section{INTRODUCTION}

In this note we focus on the analysis of system stability with constant real parameter uncertainty in state space models. Our main result involves new bounds on the system uncertainty that guarantee that the perturbed system remains stable. The literature on this problem is extensive and no attempt will be made here to give a comprehensive overview.

One of the most widely studied approaches to this problem is based upon Lyapunov stability theory. The existing bounds derived by Patel and Toda [1], Yedavalli [2], Zhou and Khargonekar [3], and Bernstein and Haddad [4], [5], which are all based on Lyapunov stability theory, actually guarantee quadratic stability which is stronger than robust stability. One advantage of quadratic stability is that it applies to timevarying uncertainty [6]. Unfortunately, however, quadratic stability is often conservative if the uncertain parameters are known to be real and constant.

In this note we derive new robust stability bounds for linear statespace systems with constant parameter uncertainty by applying a guardian map [7]-[9] to the uncertain matrices. This approach is closely related to the technique used in [10], while the robustness bounds we obtain are analogous to the bounds obtained in [3]. We show by example that the new bounds may yield a larger robust stability region than the Lyapunov bounds in [3], [4] for the case of constant uncertain parameters.

Manuscript received March 21, 1992; revised November 17, 1992. This work was partially supported by the National Science Foundation under Grant 8722244 and the Air Force Office of Scientific Research under Grant F4962092-J-0127.

The authors are with the Department of Aerospace Engineering, The University of Michigan, Ann Arbor, MI 48109-2140.

IEEE Log Number 9212849.

\section{Definttions, Notation, AND a Necessaryand} SUFFICIENT CONDITION FOR ROBUST STABILITY

Consider the uncertain linear dynamic system

$$
\dot{x}(t)=\left(A_{0}+\Delta A\right) x(t)
$$

where $A_{0}$ is an $n \times n$ real Hurwitz matrix, i.e., all the eigenvalues of $A_{0}$ have negative real part, and $\Delta A$ is a constant uncertainty matrix with the structure

$$
\Delta A=\sum_{i=1}^{p} q_{i} A_{i}
$$

where $A_{i}$ are given constant $n \times n$ real matrices, and $q_{i}$ are real constant uncertain parameters. Let $\Omega$ denote the assumed uncertainty set, that is,

$$
q \equiv\left[q_{1} q_{2} \cdots q_{p}\right]^{T} \in \Omega \subset R^{P} .
$$

We assume that $0 \in \Omega$ and that $\Omega$ is continuously arcwise connected. We say that the system (2.1) is robustly stable if $A_{0}+\Delta A$ is Hurwitz for all $\Delta A$ given by (2.2), (2.3), that is, if the matrix $A(q) \equiv A_{0}+\sum_{i=1}^{p} q_{i} A_{i}$ is Hurwitz for all $q \in \Omega$. The transformation of the original stability problem into a nonsingularity problem is based on Kronecker matrix algebra. The Kronecker product and sum operations $\otimes$ and $\oplus$ are defined in [11]. The operator vec $(F)$ stacks the columns of $F$ into a vector, while its inverse mat $(\operatorname{vec}(F))=F$ re-forms the matrix $F$ from vec $(F)$.

For $A \in R^{n \times n}$ the Lyapunov operator $L_{A}: R^{n \times n} \rightarrow R^{n \times n}$ defined by

$$
L_{A}(P):=A^{T} P+P A
$$

has the representation

$$
\operatorname{vec} L_{A}(P)=(A \oplus A)^{T} \operatorname{vec} P \text {. }
$$

Letting spec $(\cdot)$ denote spectrum, from [11] it follows that

$$
\begin{aligned}
& \operatorname{spec}(A \otimes A)=\left\{\lambda_{i} \lambda_{j}: \lambda_{i}, \lambda_{j} \in \operatorname{spec}(A)\right\}, \\
& \operatorname{spec}(A \oplus A)=\left\{\lambda_{i}+\lambda_{j}: \lambda_{i}, \lambda_{j} \in \operatorname{spec}(A)\right\} .
\end{aligned}
$$

Hence, the matrix $A \oplus A$ is nonsingular if and only if $\lambda_{i}+\lambda_{j} \neq 0$, $i, j=1,2, \cdots, n$. It thus follows that $\left(A_{0}+\Delta A\right) \oplus\left(A_{0}+\Delta A\right)$ is singular if and only if $\Delta A$ is such that $A_{0}+\Delta A$ has an eigenvalue on the imaginary axis. Thus, $\nu(A) \triangleq \operatorname{det}(A \oplus A)$ is a guardian map for the open left half plane in the sense of [7]-[9]. The following result is basic to our approach

Proposition 2.1: The system (2.1) is robustly stable if and only if

$$
\operatorname{det}\left(A_{0} \oplus A_{0}+\sum_{i=1}^{p} q_{i}\left(A_{i} \oplus A_{i}\right)\right) \neq 0, \quad \in \Omega .
$$

Proof: To prove necessity, write (2.6) as

$$
\operatorname{det}(A(q) \oplus A(q)) \neq 0, q \in \Omega \text {. }
$$

If (2.1) is robustly stable then $\operatorname{Re} \lambda_{i}(A(q) \oplus A(q))=\operatorname{Re}\left(\lambda_{j}(A(q))+\right.$ $\lambda_{k}(A(q))<0$ for every eigenvalue $\lambda_{i}$ of $A(q) \oplus A(q)$. Hence, (2.6) holds. To prove sufficiency, suppose that system (2.1) is not robustly stable, i.e., there exists $\bar{q} \in \Omega$ such that $A(\bar{q})$ is not Hurwitz. Since $A_{0}=A(0)$ is Hurwitz and $\Omega$ is continuously arcwise connected, there exists $q^{*}=\left[q_{1}^{*}, \cdots, q_{p}^{*}\right]^{T} \in \Omega$ such that $A\left(q^{*}\right)$ has a purely imaginary eigenvalue. Hence,

$$
\operatorname{det}\left(A\left(q^{*}\right) \oplus A\left(q^{*}\right)\right)=\operatorname{det}\left(A_{0} \oplus A_{0}+\sum_{i=1}^{p} q_{i}^{*}\left(A_{i} \oplus A_{i}\right)\right)=0,
$$

as required. 


\section{Robust Stability Bounds}

In this section, we use Proposition 2.1 to derive new robust stability bounds. These new bounds are based upon sufficient conditions that imply that the guardian map does not vanish.

We begin with some preliminary lemmas. A symmetric matrix $M \in R^{k \times k}$ is positive definite $(M>0)$ if $x^{T} M x$ is positive for all nonzero $x \in R^{k}$. For (arbitrary) $M \in R^{k \times k}$ we define the symmetric part of $M$ by

$$
M^{S} \triangleq \frac{1}{2}\left(M+M^{T}\right) .
$$

The following result is the basis for our approach.

Lemma 3.1: Let $M \in R^{k \times k}$. If $M^{S}>0$ then $-M$ is Hurwitz and thus $M$ is nonsingular.

Proof: If $M^{S}>0$ then $-M+(-M)^{T}<0$, which implies that $-M$ is Hurwitz. Consequently, $M$ is nonsingular.

As an application of Lemma 3.1 we have the following result.

Lemma 3.2: Let $A_{0} \oplus A_{0}$ have the singular value decomposition $A_{0} \oplus A_{0}=U \sum V$, where $U$ and $V$ are orthogonal and $\sum$ is positive diagonal, let positive diagonal $\sum_{1}, \sum_{2}$ satisfy $\sum=\sum_{1} \Sigma_{2}$, and define

$$
M_{i} \triangleq \Sigma_{1}^{-1} U^{T}\left(A_{i} \oplus A_{i}\right) V^{T} \sum_{2}^{-1}, \quad i=1, \cdots, p .
$$

If

$$
I+\sum_{i=1}^{p} q_{i} M_{i}^{s}>0, \quad q \in \Omega,
$$

then $(2.1)$ is robustly stable.

Proof: First note that (3.1) is equivalent to

$$
\left(I+\sum_{i=1}^{p} q_{i} M_{i}\right)^{S}>0, \quad q \in \Omega,
$$

which, by Lemma 3.1, implies that $I+\sum_{i=1}^{p} q_{i} M_{i}$ is nonsingular, $q \in \Omega$. Since

$$
I+\sum_{i=1}^{p} q_{i} M_{i}=\Sigma_{1}^{-1} U^{T}\left[A_{0} \oplus A_{0}+\sum_{i=1}^{p} q_{i}\left(A_{i} \oplus A_{i}\right)\right] V^{T} \Sigma_{2}^{-1}
$$

it follows that $A_{0} \oplus A_{0}+\sum_{i=1}^{p} q_{i}\left(A_{i} \oplus A_{i}\right)$ is nonsingular for all $q \in \Omega$. Proposition 2.1 thus implies that $(2.1)$ is robustly stable. $\square$

We now turn to our principal result on robust stability bounds. For this result define the following notation. If $M \in R^{n \times n}$ then $|M| \triangleq\left[\mid M_{i j}\right]_{i, j=1}^{n}, \sigma_{\max }(M)$ is the maximum singular value of $M$, and abs $(M)$ is the nonnegative-definite square root of $M M^{T}$.

Theorem 3.1: Let $M_{i}, i=1, \cdots, p$, be defined as in Lemma 3.2, and define $M_{e} \triangleq\left[M_{1}^{S} \cdots M_{p}^{S}\right]$. Then (2.1) is robustly stable for $\Omega$ defined by each of the following conditions:

$$
\sum_{i=1}^{p} q_{i}^{2}<\frac{1}{\sigma_{\max }^{2}\left(M_{\epsilon}\right)}
$$

2.

$$
\sum_{i=1}^{p}\left|q_{i}\right| \sigma_{\max }\left(M_{i}^{s}\right)<1
$$

3.

$$
\left|q_{i}\right|<\frac{1}{\sigma_{\max }\left(\sum_{i=1}^{p}\left|M_{i}^{s}\right|\right)} . \quad i=1,2, \cdots, p
$$

$$
\left|q_{i}\right|<\frac{1}{\sigma_{\max }\left(\sum_{i=1}^{p} \operatorname{abs}\left(M_{i}^{s}\right)\right)}, \quad i=1,2, \cdots, p .
$$

Proof: The results are directly analogous to the bounds given in [3] and are proved in a similar manner. For details, see [11].

Note that bound i) corresponds to a circular region, bound ii) corresponds to a diamond-shaped region, and bounds iii) and iv) are rectangular regions. As will be seen, the tightness of the bounds depends on the factorization $\sum=\sum_{1} \sum_{2}$. This will be illustrated by examples in Section IV.

In applying Theorem 3.1 to the examples in Section IV we chose the factorization $\sum=\sum_{1} \sum_{2}$ in Lemma 3.2 to be $\sum_{1}=\sum^{\alpha}$, $\sum_{2}=\sum^{1-\alpha}$, where $0 \leq \alpha \leq 1$. That is, each diagonal element $\sum_{(i)}$ of $\sum$ was factored as the product of two positive numbers between 1 and $\sum_{(i)}$. One could also allow $\alpha$ to be an arbitrary real number or choose a different value of $\alpha$ for each diagonal element of $\sum$. However, our simple factorization seemed to be adequate for the examples considered. The presence of free "balance" parameters in Lyapunov bounds is a common feature of robustness theory [5].

Finally, it can be seen that when there is a single uncertain parameter $(p=1)$ all four of the regions i)-iv) coincide.

The concept of guardian map for Hurwitz stability can be extended to more general regions in the complex plane [8]. Robustness bounds can also be derived for such regions, although the details are somewhat more complicated [12].

\section{EXAMPLES}

We first consider system (2.1) with

$$
A(q)=\left[\begin{array}{rrr}
-2 & 0 & -1 \\
0 & -3 & 0 \\
-1 & -1 & -4
\end{array}\right]+q_{1}\left[\begin{array}{lll}
1 & 0 & 1 \\
0 & 0 & 0 \\
1 & 0 & 1
\end{array}\right]+q_{2}\left[\begin{array}{lll}
0 & 0 & 0 \\
0 & 1 & 0 \\
0 & 1 & 0
\end{array}\right] .
$$

This example has been treated in [3], [4], [13]. The exact robust stability region for this problem is $\left\{\left(q_{1}, q_{2}\right): q_{1}<1.75\right.$ and $\left.q_{2}<3\right\}$. Note that the nominal parameter values $q_{1}=q_{2}=0$ are close to the boundary of the stability region. Since all of the regions given by Theorem 3.1 are symmetric with respect to the origin, it follows that the size of these regions will be severely limited. Although it is a simple matter to shift the nominal point to obtain larger regions, we shall not do so in order to remain consistent with [3], [4], [13].

Using the factorization $\sum=\sum_{1} \sum_{2}=\sum^{\alpha} \sum^{1-\alpha}, 0 \leq \alpha \leq 1$, discussed in Section III, we obtain the following regions from Theorem 3.1:

1. $q_{1}^{2}+q_{2}^{2} \leq(1.7443)^{2}$,

2. $(q 1 / 1.7465)+(q 2 / 2.7193)<1$.

3. $\left|q_{i}\right|<1.1964, i=1,2$,

4. $\left|q_{i}\right|<1.7149, i=1,2$.

Fig. 1 illustrates the search for the maximal circle radius with $0 \leq \alpha \leq 1$. In this case the largest circle was found for $\alpha=.55$. These results compare favorably with those obtained in [3], [4].

Next we consider the robust stability properties of the LQG and robustified LQG (RLQG) controllers that were analyzed in [4]. This problem involves an uncertain plant multiplicative gain parameter $\sigma_{1}$ which we denote by $q_{1}$. In [4] the robust stability region for the LQG controller was given as $-.07<q_{1}<.01$, while the robust stability region for the RLQG controller was $-.28<q_{1}<.21$. Applying Theorem 3.1 with the factorization discussed in Section III we obtained $\left|q_{1}\right|<.0093$ for the LQG controller and $\left|q_{1}\right|<.1729$ for the RLQG controller. By comparison, for the LQG controller the largest robust stability region obtained in [4] was $\left|q_{1}\right|<.00242$, while for the RLQG controller the largest robust stability region was $-.0115<q_{1}<.057$. Thus for a symmetric robust stability region the LQG prediction represents an improvement by a factor of 38 , while for the RLQG controller the improvement is by a factor of 15 . 


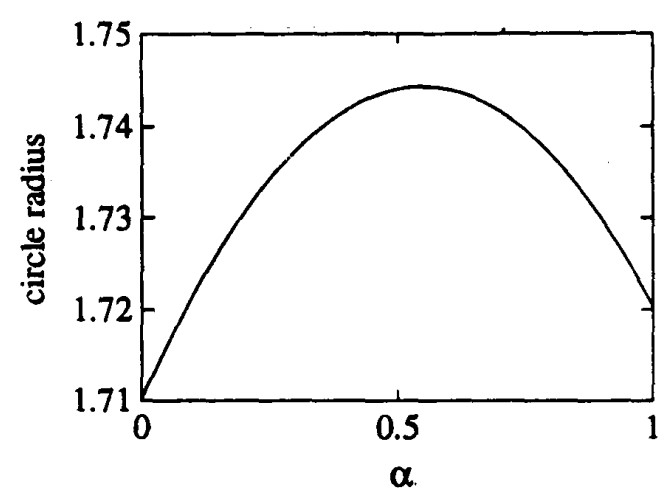

Fig. 1. Circle radius versus $\alpha$.

\section{CONCLUSION}

New bounds for robust stability have been obtained by means of guardian maps. As shown by examples in [12], these bounds apply only to constant real parameter uncertainty and thus do not imply quadratic stability. Examples were given to show improvement over Lyapunov-based bounds for constant real parameter uncertainty. The new bounds, however, may entail greater computational effort than other bounds. Furthermore, although the new bounds showed improvement over other bounds for specific examples, the new bounds may not perform as well in other cases.

\section{ACKNOWLEDGMENT}

The authors wish to thank Brian Reckamp for performing the numerical calculations reported in Section IV.

\section{REFERENCES}

[1] R. V. Patel and M. Toda, "Quantitative measures of robustness for muitivariable systems," in Proc. Joint Automat. Contr. Conf., San Francisco, CA, 1980.

[2] R. K. Yedavalli, "Improved measures of stability robustness for linear state space models," IEEE Trans. Automat. Contr., vol. AC-30, pp. $557-579,1985$

[3] K. Zhou and P. P. Khargonekar, "Stability robustness bounds for statespace models with structured uncertainty," IEEE Trans. Automat. Contr. vol. AC-32, 1987.

[4] D. S. Bernstein and W. M. Haddad, "Robust stability and performance analysis for linear dynamic systems," IEEE Trans. Automat. Contr., vol. AC-34, pp. 751-758, 1989.

[5] "Robust stability and performance analysis for state space systems via quadratic Lyapunov Bounds," SIAM J. Matrix Anal. Appl., vol. 11, pp. 239-271, 1990.

[6] B. R. Barmish, "Necessary and sufficient conditions for stabilizability of an uncertain system," J. Optmiz. Theory Appl., vol. 46, pp. 399-408, 1985.

[7] A. Tesi and A. Vicino, "Robustness analysis for uncertain dynamical systems with structured perturbations," in Proc. 27th CDC, Austin, TX, Dec. 1988.

[8] L. Saydy, A. L. Tits, and E. H. Abed, "Robust stability of linear systems relative to guarded domains," in Proc. Conf. Decision Contr., pp. 544-551, 1988.

[9] "Guardian maps and the generalized stability of parametrized families of matrices and polynomials," Math. Contr. Sig. Syst., vol. 3, pp. 345-371, 1990.

[10] M. Fu and B. R. Barmish, "Maximal unidirectional perturbation bounds for stability of polynomials and matrices," Syst. Contr. Lett., vol. 11 pp. 173-179, 1988.
[11] J. W. Brewer, "Kronecker products and matrix calculus in system theory," IEEE Trans. Circ. Syst., vol. CAS-25, pp. 772-781, 1978.

[12] S. Rern, "Robust design of state space systems with parametric uncertainties: A multimodel guaranteed cost approach," Ph.D. dissertation, Dept. Aerospace Engineering, The Univ. of Michigan, 1991.

[13] D. D. Siljak, "Parameter space methods for robust control design: A guided tour," IEEE Trans. Automat. Contr., vol. AC-34, pp. 674-688, 1989

\section{On Stability Robustness of a Dual-Rate Control System}

Tongwen Chen

\begin{abstract}
This note shows that robust stabilization of a multirate sampled-data system which has a slow sampling rate and a fast control rate can be solved via discrete-time $\mathcal{H}_{\infty}$ optimization.
\end{abstract}

\section{INTRODUCTION}

A recent trend in treating sampled-data systems is to take into account of intersample behavior. This brought solutions to the sampleddata $\mathcal{H}_{\infty}$ control problem [9], [15], [1], [14] and stability robustness problems [5], [13], all in the single-rate setting.

In [5] stability of a single-rate sampled-data system is defined in terms of continuous signals. This provides a natural framework to study stability robustness under perturbations in the analog plant. It is shown [5] that robust stabilization of such a sampled-data system can be reformulated as exactly a discrete-time $\mathcal{H}_{\infty}$ control problem and therefore be solved by known techniques.

One way to improve performance of a sampled-data system is to sample faster. However, due to various physical constraints, one may not be able to sample the measured signal as fast as one wishes; an alternative, as proposed in [6], is to use a faster rate in the D/A conversion. This motivates the study of dual-rate sampled-data systems. A similar dual-rate scheme for adaptive Smith predictors is investigated in [8]

The purpose of this note is to extend the robust stabilization result to dual-rate sampled-data systems via the powerful lifting technique [10]. Although the system in consideration has a slow sampling rate and a fast control rate, the method used in the derivation is suggestive in treating more general multirate systems.

The organization of the note is as follows. The next section presents the continuous framework for stability; Section III contains the main result on robust stabilization; and Section IV addresses some computational aspects.

The notation is quite standard: $\mathcal{C}$ is the complex plane; $\mathcal{D} \subset \mathcal{C}$ is the open unit disk; $l$ is the space of all sequences, perhaps vectorvalued, defined on $\{0,1,2, \cdots\} ; l_{2}$ is the square-summable subspace of $l ; \mathcal{L}_{2}$ is the Lebesgue space defined on $[0, \infty)$; and $\mathcal{H}_{\infty}(\mathcal{D})$ is the Hardy space defined on $\mathcal{D}$. In discrete time, we use $\lambda$-transforms instead of $z$-transforms, where $\lambda=z^{-1}$. Finally, if a linear discrete system $G$ has a state-space realization $(A, B, C, D)$, then we denote

Manuscript received March 12, 1992; revised November 17, 1992. This work was supported in part by the Natural Sciences and Engineering Research Council of Canada.

The author is with the Department of Electrical \& Computer Engineering, University of Calgary, Calgary, Alberta, Canada T2N 1N4.

IEEE Log Number 9212850 